\section{Autophagie et système cardiovasculaire}

\author{
Marouane Kheloufi ${ }^{1,2,4}$, Pierre-Emmanuel Rautou ${ }^{1-3}$, \\ Chantal M. Boulanger ${ }^{1,2}$
}

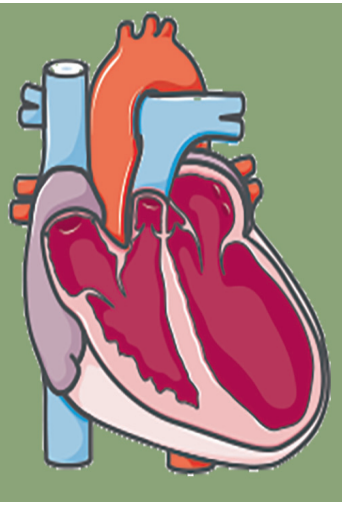

${ }^{1}$ Inserm, U970, Paris

cardiovascular research center -

PARCC, 56, rue Leblanc,

75015 Paris, France ;

2Université Paris Descartes,

Sorbonne Paris Cité, UMR-S 970 ,

Paris, France ;

${ }^{3}$ Service d'hépatologie,

DHU unity, Hôpital Beaujon,

Assistance Publique-Hôpitaux de

Paris, Clichy, France ;

${ }^{4}$ Université Denis Diderot-Paris

7, Sorbonne Paris Cité,

75018 Paris, France.

L'autophagie joue un rôle majeur dans chantal.boulanger@inserm.fr

le maintien de l'homéostasie cel-

lulaire, car elle permet l'élimination et le remplacement continuel des protéines et des organites non fonctionnels. Elle est induite par différents stress cellulaires (hypoxie, stress oxydant, carences en nutriments, infections virales) et agit généralement comme un mécanisme protecteur qui va promouvoir la survie de la cellule au sein d'un environnement défavorable. Néanmoins, un excès d'autophagie, aboutissant à la destruction d'une forte proportion du cytoplasme, peut conduire à la mort cellulaire. On parle alors de mort autophagique (ou mort cellulaire de type 2) ou autose [2].

Des efforts de recherche considérables ont été mis en œuvre afin de mieux comprendre le rôle de l'autophagie en physiopathologie cardiovasculaire et pour identifier de nouvelles cibles thérapeutiques potentielles. Toutes les cellules du système cardiovasculaire présentent des capacités d'autophagie et de nombreuses études montrent l'implication du processus dans le développement et la progression des maladies cardiovasculaires. Cette synthèse a pour but de discuter les études récentes ayant évalué le rôle de l'autophagie dans ces maladies.

\section{Autophagie et maladies cardiaques}

\section{Autophagie et vieillissement cardiaque}

Plusieurs études mettent en évidence un rôle essentiel de l'autophagie dans le maintien de l'homéostasie cardiaque au cours du vieillissement. L'autophagie cardiaque décroît spontanément avec l'âge. Une altération de la voie autophagique par déficience d'Atg5 (autophagyrelated protein 5 ), spécifiquement dans les cardiomyocytes, entraîne une cardiomyopathie hypertrophique caractérisée par une hypertro- 
phie ventriculaire gauche et une diminution de la fraction de raccourcissement ${ }^{1}$ chez des souris transgéniques âgées [3]. L'inhibition de l'autophagie par suractivation de la voie mTOR (mammalian target of rapamycin) liée à la déficience en GSK3a (glycogen synthase kinase 3 alpha) avec l'âge induit une hypertrophie cardiaque et un dysfonctionnement contractile [4]. Dans le cœur des souris âgées, l'inhibition du flux autophagique par la chloroquine aboutit à l'accumulation de dépôts de lipofuscine ${ }^{2}$ [5]. À l'inverse, l'activation de l'autophagie par inhibition de mTOR prévient la dysfonction ainsi que la sénescence du tissu cardiaque $[4,5]$. De même, l'inhibition de la sous-unité catalytique pl10 $\alpha$ de la PI3K (phosphoinositide 3-kinase), un activateur de mTOR, induit l'autophagie et aboutit aussi à une préservation de la fonction cardiaque, une diminution de la sénescence, ainsi que l'accumulation des dépôts de lipofuscine [5].

Le déclin progressif de la fonction mitochondriale des cardiomyocytes est considéré comme l'un des mécanismes principaux de la dysfonction cardiaque liée à l'âge [6]. Les mitochondries dysfonctionnelles produisent moins d'ATP (adénosine triphosphate) et génèrent plus d'espèces réactives de l'oxygène [6]. L'autophagie régule la fonction mitochondriale en dégradant les mitochondries altérées par un processus appelé mitophagie [57] $(\rightarrow)$.

$\rightarrow$ Voir la Synthèse de P. Vigié et $\mathbf{N}$. Camougrand, page 231 de ce numéro

La mitophagie est altérée par la perturbation que p53 induit sur la translocation de Parkin ${ }^{3}$. La surexpression de Parkin, ainsi que la déficience en p53, préviennent la sénescence cellulaire et le déclin de la fonction cardiaque au cours du vieillissement $[7,8]$. L'activation de la mitophagie par surexpression de la HSP27 (heat shock protein 27), une molécule impliquée dans la réponse au stress, atténue le déclin de la fonction cardiaque en diminuant la sénescence, la fibrose ainsi que le stress oxydant cardiaque [9].

\section{Rôles de l'autophagie pendant l'ischémie/reperfusion}

Au cours de l'ischémie cardiaque, l'apport en oxygène et nutriments aux cardiomyocytes est fortement diminué. Le processus autophagique est alors activé afin de réapprovisionner les cellules en substrats métaboliques essentiels et d'éliminer les organelles endommagées, particulièrement les mitochondries, sources importantes d'espèces réactives de l'oxygène et de médiateurs pro-apoptotiques comme le cytochrome C [10]. L'activité autophagique au cours d'un épisode ischémique aigu semble résulter de l'inhibition de mTOR dépendante de la protéine kinase activée par I'AMP (AMPK) [11]. Le rôle de l'autophagie durant la phase ischémique reste cependant controversé. Certaines études ont montré un effet pro-survie de l'autophagie [12], alors que d'autres concluent à un effet délétère [13]. Ces discordances pourraient s'expliquer par l'utilisation de protocoles expérimentaux différents (cultures primaires versus lignées cellulaires, temps d'ischémie différents). Dans un modèle d'ischémie chronique, un rôle protecteur de l'autophagie a été suggéré : le niveau d'expres-

\footnotetext{
${ }^{1}$ Rapport de la différence des diamètres télédiastolique et télésystolique sur le diamètre télédiastolique. C'est un indice de la fonction ventriculaire gauche.

${ }^{2}$ Pigment qui résulte de la dégradation des organites.

${ }^{3}$ Parkin est une ubiquitine ligase dont le déficit est associé à la maladie de Parkinson.
}

sion des marqueurs de l'autophagie est en effet inversement associé au degré de mort cellulaire [14]. L'effet cardioprotecteur du préconditionnement ischémique semble également nécessiter l'activation de la cascade autophagique [15]. L'ensemble de ces études plaide donc en faveur d'un rôle protecteur de l'autophagie au cours de l'ischémie cardiaque.

Pendant la reperfusion, en dépit du réapprovisionnement en oxygène et nutriments, l'activité autophagique est considérablement augmentée. Cet effet résulte d'une augmentation de l'activité de Beclin-l (une des protéines autophagiques), indépendamment de l'AMPK [11]. Cependant, cette activation semble être inefficace en raison d'un défaut d'élimination des autophagosomes excessivement générés [16], peut-être dû à une diminution de l'expression de LAMP-2 (lysosomalassociated membrane protein-2), une protéine importante dans le processus de fusion des lysosomes avec les autophagosomes [17].

\section{Autophagie et insuffisance cardiaque}

L'insuffisance cardiaque est une maladie chronique caractérisée par un remodelage ventriculaire et une perte progressive de cardiomyocytes aboutissant à une dysfonction contractile. L'analyse d'échantillons de patients insuffisants cardiaques met en évidence la survenue d'une mort autophagique [18], suggérant que l'autophagie aurait un rôle délétère. Cette hypothèse d'un rôle nocif de l'autophagie dans l'insuffisance cardiaque est confortée par plusieurs observations. Zhu et ses collaborateurs ont ainsi montré que l'insuffisance cardiaque par surcharge de pression s'accompagnait d'une augmentation de l'activité autophagique. L'haplo-insuffisance ${ }^{4}$ en Beclin-1 diminue l'induction de l'autophagie et prévient partiellement l'insuffisance cardiaque. À l'inverse, la surexpression de la protéine au niveau cardiaque accentue le remodelage pathologique [19]. Dans ce même modèle, l'acétyl-coenzyme A, en inhibant l'autophagie, prévient le développement de l'hypertrophie cardiaque [20]. Par ailleurs, les inhibiteurs de $\mathrm{HDACl} / 2$ (histone deacétylases) qui suppriment l'induction du flux autophagique, empêchent le remodelage cardiaque [21], soulignant ainsi l'importance de l'acétylation de certaines protéines de la cascade autophagique dans ce processus inadapté.

La déficience cardiaque en Atg5 (autophagy-related gene 5) qui code l'une des protéines autophagiques, se traduit par une hypertrophie cardiaque, une dilatation ventriculaire ainsi qu'une dysfonction contractile [22]. L'autophagie basale semble donc importante pour le

\footnotetext{
${ }^{4}$ Qui se traduit par une perte d'expression.
} 
maintien de la balance entre synthèse et dégradation protéique au niveau cardiaque. Dans un modèle murin de cardiopathie liée à la desmine ${ }^{5}$, la surexpression d'Atg7 améliore la fonction contractile, réduit l'hypertrophie et prolonge la survie [23]. De même, la rapamycine, un puissant activateur de l'autophagie, prévient l'hypertrophie cardiaque induite par les hormones thyroïdiennes [24], réduit I'hypertrophie cardiaque et améliore la fonction ventriculaire dans un modèle de surcharge de pression [25]. Enfin, l'activation pharmacologique de I'AMPK par la metformine ou I'AICAR (5-aminoimidazole-4-carboxamide ribonucleotide) réduit significativement l'hypertrophie cardiaque en augmentant l'activité autophagique via l'inhibition de mTORCl (mammalian target of rapamycin complex 1) [26]. L'autophagie basale apparaît donc protectrice au cours de l'insuffisance cardiaque. Cependant à la suite d'un stress, l'excès d'autophagie devient inadapté et délétère.

Les résultats de nombreuses études restent difficiles à interpréter en raison de l'absence d'évaluation du flux autophagique. En effet, l'augmentation du nombre d'autophagosomes peut résulter soit d'un accroissement de leur synthèse, soit d'une diminution de leur élimination [27]. Ainsi, dans le modèle murin déficient en LAMP-2, les animaux présentent une contractilité cardiaque sévèrement réduite, ainsi que des cardiomyocytes d'ultrastructure anormale avec une accumulation d'autophagosomes [28]. De même, chez l'homme, les cardiomyocytes différenciés à partir de fibroblastes issus de patients atteints de la maladie de Danon (une affection génétique rare liée au chromosome $X$ caractérisée par une cardiomyopathie hypertrophique sévère, causée par une mutation du même gène $L A M P-2)$ reprogrammés en cellules souches pluripotentes, ont un blocage du flux autophagique, associé à une augmentation de la taille cellulaire, du stress oxydatif mitochondrial ainsi que de l'apoptose [29].

\section{Autophagie et cardiomyopathie diabétique}

Dans des modèles murins de diabète de type 1 (diabète induit par I'injection de streptozotocine ou souris diabétiques OVદ26), I'autophagie cardiaque est inhibée [30], le flux autophagique réduit [31] et les niveaux d'expression des marqueurs de la mitophagie PINK-1 (PTEN-induced putative kinase 1), Parkin et BNIP3 (BCL2/adenovirus $\varepsilon 1 B 19 k D a$ interacting protein 3) sont abaissés. De manière intéressante, chez les souris diabétiques dont l'expression de Beclin-1 ou Atgl6 est altérée, la dysfonction cardiaque est également significativement réduite, tandis que la surexpression de Beclin-1 conduit à l'effet inverse. La baisse du niveau d'autophagie et de la mitophagie apparaît donc comme une réponse adaptative au diabète [31], malgré une dysfonction mitochondriale importante. Le traitement chronique par la metformine (qui active l'autophagie via la voie AMPK et est utilisée dans le traitement du diabète) améliore cependant la fonction cardiaque dans ces modèles [30]. Ce résultat bénéfique de la metformine pourrait s'expliquer par des effets indépendants de l'autophagie.

${ }^{5}$ La desmine relie les fibres musculaires au niveau des stries $Z$ et assure la cohésion cellulaire des disques intercalaires cardiaques. L'absence de desmine fonctionnelle provoque une cardiomyopathie et des défauts vasculaires.
Dans des modèles de diabète de type 2, les résultats des études ayant exploré l'autophagie cardiaque sont contradictoires. En effet, dans un modèle de syndrome métabolique induit par un régime riche en lipides, une inhibition de l'autophagie, associée à une cardiomyopathie hypertrophique, est observée, le traitement par la rapamycine, qui inhibe mTOR, atténuant ces effets [32]. Cependant, dans le modèle d'insulinorésistance induite par un régime riche en fructose, la perte de cardiomyocytes ainsi que la fibrose cardiaque sont associées à une activation de l'autophagie résultant de la suppression de la signalisation Akt (ou protéine kinase B) [33]. Enfin, dans un modèle murin de régime riche en lipides de lait, l'hypertrophie cardiaque dépend de l'activation du processus autophagique [34]. Ces divergences pourraient s'expliquer par les différences de composition des régimes utilisés et les durées des protocoles expérimentaux. Comme c'est le cas pour d'autres études (voir plus haut), l'importance de l'autophagie ne peut être appréciée correctement dans ces modèles en raison de l'absence d'évaluation du flux autophagique. Xu et ses collaborateurs ont cependant montré le rôle fonctionnel de l'autophagie dans un modèle d'obésité induite par un régime riche en lipides, dans lequel la cardiomyopathie diabétique s'accompagne d'une accumulation de vacuoles autophagiques en raison d'un blocage du flux autophagique [35].

\section{Autophagie et maladies vasculaires}

\section{Autophagie et athérosclérose}

La mise en évidence de structures autophagiques au sein des plaques d'athérosclérose, à la fois au niveau des cellules endothéliales, musculaires lisses et des macrophages [36], suggère que l'autophagie joue un rôle important dans le développement de la maladie athéromateuse (Figure 1). Plusieurs facteurs pro-athérogènes stimulent en effet l'autophagie comme les lipoprotéines de faible densité (LDL) oxydées [37], le TNF- $\alpha$ (tumor necrosis factor alpha) [38] ou encore le 7-kétocholestérol [39].

L'autophagie est impliquée dans la conversion des macrophages en cellules spumeuses ${ }^{6}$ et dans le développement de la plaque d'athérosclérose. Elle est nécessaire à l'efflux du cholestérol des cellules spumeuses en favorisant la lipolyse lysosomale des gouttelettes lipidiques [40]. L'inhibition pharmacologique, ou moléculaire, de l'autophagie réduit en effet significativement l'efflux du cholestérol [40]. Sa suppression par l'activation de mTOR, favorise la formation des

\footnotetext{
${ }^{6}$ Macrophages chargés de gouttelettes de lipides.
} 


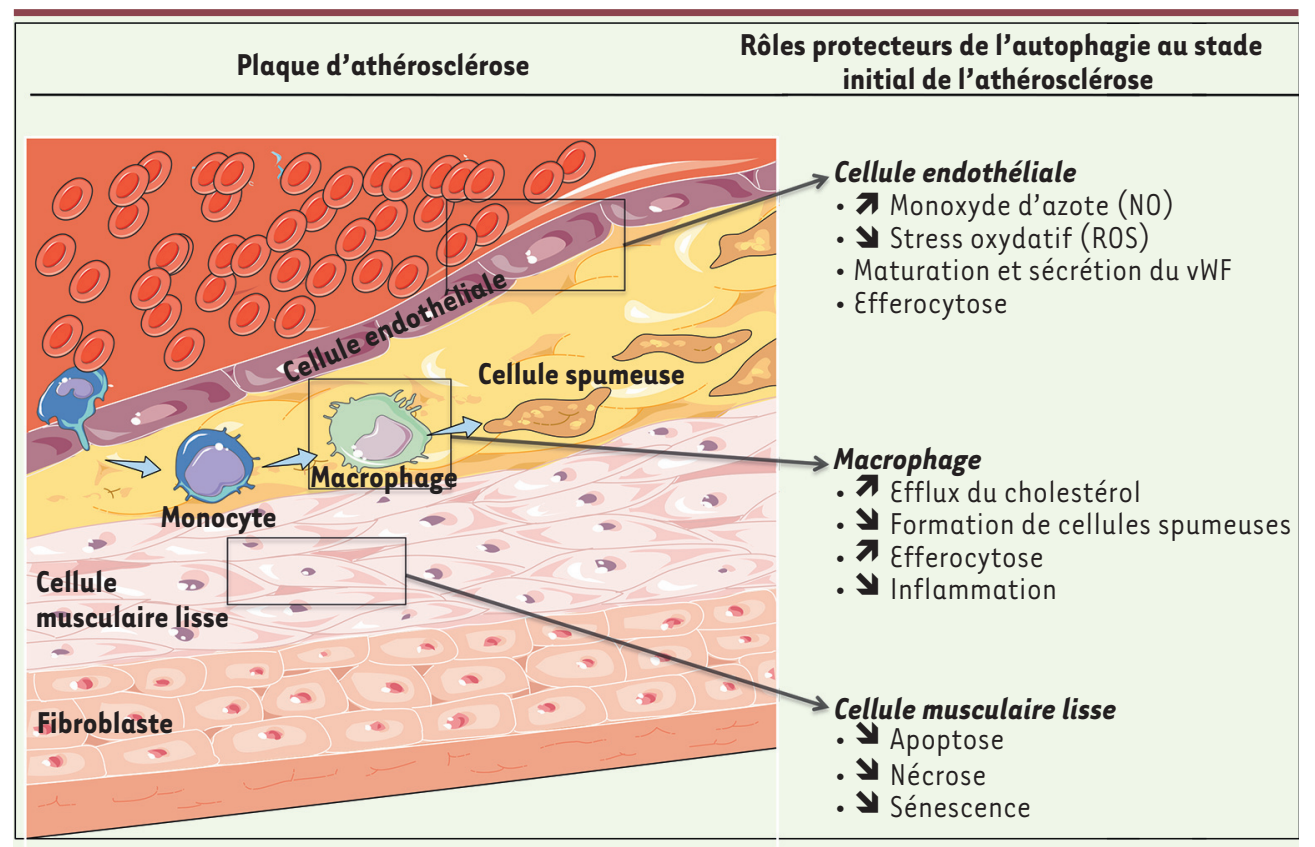

Figure 1. Rôle de l'autophagie au cours des stades initiaux du développement de l'athérosclérose. ROS : reactive oxygen species; vWF : facteur von Willebrand.

Les effets pro-sénescents et antioxydants du défaut d'autophagie auraient pour origine l'accumulation de p62 (séquestosome 1) [46]. L'autophagie peut donc présenter des rôles différents selon le type cellulaire concerné. Plusieurs fonctions essentielles au maintien de l'homéostasie endothéliale sont régulées par l'auto-

cellules spumeuses [41]. De même, la délétion de Wip-1 (p53-induced phosphatase), un régulateur négatif de la voie impliquant ATM (ataxia-telangiectasia mutated) et mTOR, conduit à l'activation de l'autophagie, l'inhibition de la conversion des macrophages en cellules spumeuses et à la prévention de la formation des plaques [42]. De récentes études ont montré un rôle protecteur de l'activité autophagique des macrophages dans l'athérosclérose. En effet, la déficience en autophagie uniquement dans les macrophages exacerbe le développement des plaques chez les souris Ldlr/-- (low density lipoprotein receptor) [43]. Chez ces souris, l'excès de production d'espèces réactives de l'oxygène aboutit à une augmentation de l'apoptose associée à un défaut d'élimination des corps apoptotiques [43]. La déficience en autophagie des macrophages s'accompagne également d'une hyperactivation de l'inflammasome et d'une augmentation de la production de l'interleukine $1 \beta$ [44].

Plusieurs travaux ont montré un rôle protecteur de l'autophagie dans les cellules musculaires lisses. L'induction de l'autophagie par le 7-kétocholestérol inhibe l'apoptose de ces cellules [39]. La rapamycine prévient également l'apoptose et la nécrose des cellules musculaires lisses surchargées en cholestérol, tandis que la 3-méthyladénine, qui inhibe l'autophagie, l'amplifie [45]. Grootaert et ses collaborateur ont montré le rôle central de l'autophagie des cellules musculaires lisses dans l'athérosclérose, dans le modèle des souris Apo $\varepsilon^{-/-}$(apolipoprotéine $\varepsilon$ ), dans lequel un déficit spécifique en $\operatorname{Atg} 7$ dans les cellules musculaires lisses, augmente le développement des plaques d'athérosclérose [46]. Le défaut d'autophagie est également associé à une augmentation de la sénescence des cellules musculaires lisses qui se caractérise par une hypertrophie nucléaire et une augmentation de l'expression de la protéine pl6 (cyclin-dependent kinase inhibitor 2 A ou multiple tumor suppressor 1). À l'inverse des macrophages, les cellules musculaires lisses déficientes en autophagie résistent au stress oxydatif en surexprimant plusieurs enzymes anti-oxydantes. phagie. En effet, le flux autophagique augmente la biodisponibilité du NO (monoxyde d'azote), réduit le stress oxydatif ainsi que l'inflammation. Des stratégies pharmacologiques visant à stimuler l'autophagie améliorent la dysfonction endothéliale liée au vieillissement [47]. Sa déficience dans l'endothélium entraîne une perturbation de maturation et de sécrétion du facteur von Willebrand $(v W F)^{7}$ avec une diminution des taux de multimères à haut poids moléculaire et un allongement du temps de saignement [48]. L'autophagie est aussi impliquée dans l'externalisation de la phosphatidylsérine, un signal de phagocytose, induite par l'exposition de cellules endothéliales à des lipoprotéines de faible densité oxydées. L'autophagie pourrait donc avoir un rôle dans le processus d'efferocytose (phagocytose spécifique) des corps apoptotiques [37].

Les mécanismes reliant l'autophagie endothéliale au développement des plaques d'athérosclérose sont encore mal compris. Cependant, la déficience en autophagie endothéliale augmente la rétention cellulaire des lipides, résultant d'une diminution de leur dégradation. Également, les souris $A p o \varepsilon^{-/-}$ayant une déficience de l'autophagie, spécifiquement dans les cellules endothéliales, développent plus de lésions athéromateuses comparées aux souris contrôles [49].

\section{Autophagie et hypertension artérielle pulmonaire} L'hypertension artérielle pulmonaire (HTAP) est une maladie progressive caractérisée par une augmentation de la pression de l'artère pulmonaire avec un remo-

\footnotetext{
${ }^{7}$ Glycoprotéine intervenant dans le cadre du processus de l'hémostase primaire.
} 


\begin{tabular}{|c|c|c|c|c|}
\hline Maladie & $\begin{array}{c}\text { Agent } \\
\text { pharmacologique }\end{array}$ & $\begin{array}{c}\text { Effet sur } \\
\text { l'autophagie }\end{array}$ & Effet sur la maladie & Référence \\
\hline \multirow{5}{*}{ Cardiaque } & Rapamycine & Active & Prévient l'hypertrophie cardiaque liée à l'âge & [4] \\
\hline & & & Prévient l'hypertrophie cardiaque & [25] \\
\hline & Metformine & Active & Améliore la fonction cardiaque chez les souris diabétiques & [26] \\
\hline & AICAR & Active & Prévient l'hypertrophie cardiaque & [25] \\
\hline & Trichostatine & Inhibe & Prévient le remodelage cardiaque pathologique & [20] \\
\hline \multirow{3}{*}{ Vasculaire } & Tréhalose & Active & Prévient le vieillissement artériel & {$[46]$} \\
\hline & Rapamycine & Active & Inhibe la progression de l'anévrisme déjà établi & [53] \\
\hline & Chloroquine & Inhibe & Retarde la progression de l'HTAP & {$[51]$} \\
\hline
\end{tabular}

Tableau I. Interventions pharmacologiques sur les mécanismes de l'autophagie dans les maladies cardiovasculaires. AICAR : 5-aminoimidazole4-carboxamide ribonucléotide; HTAP : hypertension artérielle pulmonaire.

delage hypertrophique de sa paroi et une défaillance ventriculaire droite [50]. Peu d'études ont évalué le rôle de l'autophagie dans cette pathologie. Lee et ses collaborateurs ont observé une surexpression de la protéine autophagique LC3 (microtubule-associated protein IA/IBlight chain) ainsi qu'une augmentation du rapport LC3II/I (rapport entre formes membranaire et libre de la protéine) dans les poumons de patients atteints d'HTAP et dans un modèle murin [51]. L'autophagie jouerait un rôle protecteur dans cette pathologie, les souris $L c 3 b^{-1-}$ développant plus rapidement une HTAP sous l'effet de l'hypoxie [51]. Cependant, dans un autre modèle d'HTAP induite par la monocrotaline chez le rat, l'inhibition de l'autophagie par la chloroquine atténue l'installation de la maladie et retarde sa progression, indiquant ici un rôle délétère pour l'autophagie. L'effet de la chloroquine reposerait sur l'inhibition de la dégradation lysosomale de BMPR-II (bone morphogenetic protein type II receptor), une protéine inhibant la prolifération et induisant l'apoptose des cellules musculaires lisses des artères pulmonaires [52]. À noter que ces deux modèles d'HTAP divergent quant aux mécanismes qui sont impliqués: I'HTAP induite par l'hypoxie est en effet due essentiellement à une vasoconstriction, tandis que la monocrotaline utilisée chez le rat induit une HTAP qui présente une composante inflammatoire importante.

\section{Autophagie et anévrisme de l'aorte abdominale}

L'anévrisme de l'aorte abdominale est une maladie inflammatoire de la paroi artérielle, caractérisée par une dilatation localisée de la portion abdominale de l'aorte, qui survient essentiellement chez l'homme, à partir de 60 ans. L'analyse d'échantillons humains d'anévrisme a mis en évidence une augmentation de l'expression de plusieurs gènes de la machinerie autophagique, notamment Atg4b, Beclin-1, Bnip3 (BCL2 interacting protein 3 ) et Vps34 (class III PI3K), ainsi que son activation attestée par l'augmentation du rapport LC3II/I [53]. L'ostéopontine, une cytokine pro-inflammatoire fortement exprimée au cours de l'anévrisme, est un puissant inducteur de l'autophagie in vitro. L'acti- vation de l'autophagie par la rapamycine exacerbe la mort des cellules musculaires lisses induite par l'ostéopontine, tandis que son inhibition par la 3-méthyladénine la prévient [53]. Ces observations suggèrent donc un rôle délétère de l'autophagie. Cependant, in vivo, la rapamycine inhibe la progression de l'anévrisme déjà établi [54]. L'activation de l'autophagie au niveau d'autres cellules impliquées dans le développement de l'anévrisme, notamment les cellules inflammatoires, ou des effets de la rapamycine dépendants de mTOR mais indépendants de l'autophagie, pourraient expliquer ces résultats. À noter que le traitement chronique par la chloroquine, qui inhibe la phase terminale de l'autophagie, n'a aucun impact sur le développement de la pathologie anévrismale [55].

\section{Perspectives thérapeutiques}

L'accumulation des connaissances concernant les mécanismes de régulation de l'autophagie ainsi que son rôle dans la physiopathologie cardiovasculaire permet d'envisager de cibler cette voie dans un but thérapeutique. Néanmoins, la complexité des mécanismes régulant l'autophagie ainsi que ses différents rôles dans les pathologies cardiovasculaires imposent la prise en compte de plusieurs critères avant d'envisager une intervention. Le choix de la cible dépend en effet fortement du contexte pathologique, l'autophagie pouvant être activée ou inhibée par plusieurs mécanismes différents. Outre le type cellulaire, l'intensité de modulation de l'autophagie est un autre paramètre important, ajoutant un niveau de complexité. La spécificité des agents pharmacologiques 
qui peuvent présenter des effets indépendants de l'autophagie, est également à prendre en compte. Plusieurs exemples d'interventions pharmacologiques à visée thérapeutique qui ont été développées dans des modèles animaux de maladies cardiovasculaires sont présentés dans le Tableaul.

\section{Conclusion}

L'autophagie joue un rôle indéniable dans le maintien de l'homéostasie cardiovasculaire. Des efforts de recherche considérables ont révélé des modifications du flux autophagique dans différentes pathologies cardiovasculaires. Ces modifications peuvent être adaptatives, entraînant alors une réponse bénéfique au stress, mais elles peuvent également être nuisibles, contribuant ainsi au développement ou à l'aggravation de la maladie. Cibler l'autophagie dans le traitement de ces maladies reste une approche attractive. Cependant, de nombreux travaux demeurent nécessaires afin d'identifier et de caractériser précisément dans chaque pathologie les régulateurs/effecteurs du processus autophagique, permettant ainsi de développer des stratégies thérapeutiques spécifiques et pertinentes. $\diamond$

\section{SUMMARY}

\section{Autophagy in the cardiovascular system}

Cardiovascular diseases are the leading cause of mortality worldwide. Studies regarding the role of autophagy in cardiac and vascular tissues have opened new therapeutic avenues to treat cardiovascular disorders. Altogether, these studies point out that autophagic activity needs to be maintained at an optimal level to preserve cardiovascular function. Reaching this goal constitutes a challenge for future efficient therapeutic strategies. The present review therefore highlights recent advances in the understanding of the role of autophagy in cardiovascular pathologies. $\diamond$

\section{LIENS D'INTÉRÊT}

Les auteurs déclarent n'avoir aucun lien d'intérêt concernant les données publiées dans cet article.

\section{RÉFÉRENCES}

1. Global, regional, and national life expectancy, all-cause mortality, and cause-specific mortality for 249 causes of death, 1980-2015 : a systematic analysis for the global burden of disease study 2015. Lancet $2016 ; 388: 1459-544$.

2. Codogno P, Meijer AJ. Autophagy and signaling : their role in cell survival and cell death. Cell Death Differ 2005 ; 12 (suppl 2) : 1509-18.

3. Taneike M, Yamaguchi 0 , Nakai A, et al. Inhibition of autophagy in the heart induces age-related cardiomyopathy. Autophagy $2010 ; 6: 600-6$.

4. Zhou J, Freeman TA, Ahmad F, et al. GSK-3alpha is a central regulator of age-related pathologies in mice. J Clin Invest $2013 ; 123: 1821-32$.

5. Inuzuka Y, Okuda J, Kawashima T, et al. Suppression of phosphoinositide 3-kinase prevents cardiac aging in mice. Circulation $2009 ; 120: 1695-703$.

6. Dutta D, Calvani R, Bernabei R, et al. Contribution of impaired mitochondrial autophagy to cardiac aging : mechanisms and therapeutic opportunities. Circ Res $2012 ; 110$ : 1125-38.

7. Kubli DA, Quinsay MN, Gustafsson AB. Parkin deficiency results in accumulation of abnormal mitochondria in aging myocytes. Commun Integr Biol 2013; 6 : e24511.

8. Hoshino A, Mita Y, Okawa Y, et al. Cytosolic p53 inhibits Parkin-mediated mitophagy and promotes mitochondrial dysfunction in the mouse heart. Nat Commun $2013 ; 4: 2308$.

9. Lin S, Wang $Y$, Zhang $X$, et al. HSP27 alleviates cardiac aging in mice via a mechanism involving antioxidation and mitophagy activation. Oxid Med Cell Longev 2016; 2016 : 2586706.
10. Zhang H, Bosch-Marce M, Shimoda LA, et al. Mitochondrial autophagy is an HIF-1-dependent adaptive metabolic response to hypoxia.J Biol Chem $2008 ; 283$ : 10892-903.

11. Matsui Y, Takagi H, Qu X, et al. Distinct roles of autophagy in the heart during ischemia and reperfusion: roles of AMP-activated protein kinase and Beclin 1 in mediating autophagy. Circ Res 2007 ; 100 : 914-22.

12. Kanamori H, Takemura G, Goto K, et al. Autophagy limits acute myocardial infarction induced by permanent coronary artery occlusion. Am J Physiol Heart Circ Physiol 2011 ; 300 : H2261-71.

13. Valentim L, Laurence KM, Townsend PA, et al. Urocortin inhibits Beclinlmediated autophagic cell death in cardiac myocytes exposed to ischaemia/ reperfusion injury. J Mol Cell Cardiol 2006 ; 40 : 846-52.

14. Yan L, Vatner DE, Kim SJ, et al. Autophagy in chronically ischemic myocardium. Proc Natl Acad Sci USA 2005 ; 102 : 13807-12.

15. Yan WJ, Dong HL, Xiong LZ. The protective roles of autophagy in ischemic preconditioning. Acta Pharmacol Sin 2013 ; 34 : 636-43.

16. Ma X, Liu H, Foyil SR, et al. Impaired autophagosome clearance contributes to cardiomyocyte death in ischemia/reperfusion injury. Circulation 2012 ; $125: 3170-81$.

17. Ma X, Liu H, Foyil SR, et al. Autophagy is impaired in cardiac ischemiareperfusion injury. Autophagy $2012 ; 8$ : 1394-6.

18. Kostin S, Pool L, Elsasser A, et al. Myocytes die by multiple mechanisms in failing human hearts. Circ Res $2003 ; 92: 715-24$.

19. Zhu H, Tannous P, Johnstone JL, et al. Cardiac autophagy is a maladaptive response to hemodynamic stress. J Clin Invest 2007 ; 117 : 1782-93.

20. Marino G, Pietrocola F, Eisenberg T, et al. Regulation of autophagy by cytosolic acetyl-coenzyme A. Mol Cell $2014 ; 53: 710-25$.

21. Cao DJ, Wang ZV, Battiprolu PK, et al. Histone deacetylase (HDAC) inhibitors attenuate cardiac hypertrophy by suppressing autophagy. Proc Natl Acad Sci USA 2011 ; 108 : 4123-8.

22. Nakai A, Yamaguchi 0 , Takeda T, et al. The role of autophagy in cardiomyocytes in the basal state and in response to hemodynamic stress. Nat Med 2007 ; 13 : 619-24.

23. Bhuiyan MS, Pattison JS, Osinska H, et al. Enhanced autophagy ameliorates cardiac proteinopathy. J Clin Invest 2013 ; 123 : 5284-97.

24. Kuzman JA, O'Connell TD, Gerdes AM. Rapamycin prevents thyroid hormoneinduced cardiac hypertrophy. Endocrinology $2007 ; 148$ : 3477-84.

25. Bishu K, Ogut 0 , Kushwaha $S$, et al. Anti-remodeling effects of rapamycin in experimental heart failure : dose response and interaction with angiotensin receptor blockade. PLoS One $2013 ; 8:$ e81325.

26. Li Y, Chen C, Yao F, et al. AMPK inhibits cardiac hypertrophy by promoting autophagy via mTORCl. Arch Biochem Biophys $2014 ; 558$ : 79-86.

27. Wang $X$, Robbins J. Proteasomal and lysosomal protein degradation and heart disease. J Mol Cell Cardiol 2014 ; $71: 16-24$.

28. Tanaka $Y$, Guhde $G$, Suter $A$, et al. Accumulation of autophagic vacuoles and cardiomyopathy in LAMP-2-deficient mice. Nature $2000 ; 406$ : 902-6.

29. Hashem SI, Perry CN, Bauer M, et al. Brief report : oxidative stress mediates cardiomyocyte apoptosis in a human model of Danon disease and heart failure. Stem Cells $2015 ; 33: 2343-50$.

30. Xie Z, Lau K, Eby B, et al. Improvement of cardiac functions by chronic metformin treatment is associated with enhanced cardiac autophagy in diabetic OVE26 mice. Diabetes $2011 ; 60: 1770-8$.

31. Xu X, Kobayashi S, Chen $\mathrm{K}$, et al. Diminished autophagy limits cardiac injury in mouse models of type 1 diabetes. J Biol Chem 2013; 288 : 18077-92.

32. Guo R, Zhang Y, Turdi S, Ren J. Adiponectin knockout accentuates high fat diet-induced obesity and cardiac dysfunction : role of autophagy. Biochim Biophys Acta 2013 ; 1832 : 1136-48.

33. Mellor KM, Bell JR, Young MJ, et al. Myocardial autophagy activation and suppressed survival signaling is associated with insulin resistance in fructose-fed mice. J Mol Cell Cardiol 2011 ; 50 : 1035-43.

34. Russo SB, Baicu CF, Van Laer A, et al. Ceramide synthase 5 mediates lipidinduced autophagy and hypertrophy in cardiomyocytes. J Clin Invest 2012 ; $122: 3919-30$.

35. Xu X, Hua Y, Nair S, et al. Akt2 knockout preserves cardiac function in highfat diet-induced obesity by rescuing cardiac autophagosome maturation. $J$ Mol Cell Biol $2013 ; 5: 61-3$.

36. Martinet W, De Meyer GR. Autophagy in atherosclerosis: a cell survival and death phenomenon with therapeutic potential. Circ Res 2009; $104: 304-17$.

37. Muller C, Salvayre R, Negre-Salvayre A, Vindis C. Oxidized LDLs trigger endoplasmic reticulum stress and autophagy : prevention by HDLs. Autophagy $2011 ; 7: 541-3$. 


\section{RÉFÉRENCES}

38. Jia G, Cheng G, Gangahar DM, Agrawal DK. Insulin-like growth factor-1 and TNF-alpha regulate autophagy through c-jun $\mathrm{N}$-terminal kinase and Akt pathways in human atherosclerotic vascular smooth cells. Immunol Cell Biol $2006 ; 84: 448-54$.

39. He C, Zhu H, Zhang W, et al. 7-Ketocholesterol induces autophagy in vascular smooth muscle cells through Nox4 and Atg4B. Am J Pathol 2013; 183: 626-37.

40. Ouimet M, Franklin V, Mak $\varepsilon$, et al. Autophagy regulates cholesterol efflux from macrophage foam cells via lysosomal acid lipase. Cell Metab $2011 ; 13: 655-67$

41. Wang X, Li L, Niu X, et al. mTOR enhances foam cell formation by suppressing the autophagy pathway. DNA Cell Biol 2014 ; 33 : 198-204.

42. Le Guezennec X, Brichkina A, Huang YF, et al. Wipl-dependent regulation of autophagy, obesity, and atherosclerosis. Cell Metab 2012; 16:68-80.

43. Liao X, Sluimer JC, Wang $Y$, et al. Macrophage autophagy plays a protective role in advanced atherosclerosis. Cell Metab $2012 ; 15:$ 545-53.

44. Razani $B$, Feng $C$, Coleman $T$, et al. Autophagy links inflammasomes to atherosclerotic progression. Cell Metab $2012 ; 15: 534-44$.

45. Xu K, Yang $Y$, Yan $M$, et al. Autophagy plays a protective role in free cholesterol overload-induced death of smooth muscle cells. J Lipid Res $2010 ; 51: 2581-90$.

46. Grootaert MO, da Costa Martins PA, Bitsch N, et al. Defective autophagy in vascular smooth muscle cells accelerates senescence and promotes neointima formation and atherogenesis. Autophagy 2015 ; 11 : 2014-32.

47. LaRocca TJ, Henson GD, Thorburn A, et al. Translational evidence that impaired autophagy contributes to arterial ageing. J Physiol 2012; 590 : 3305-16.

48. Torisu $T$, Torisu $K$, Lee IH, et al. Autophagy regulates endothelial cell processing, maturation and secretion of von Willebrand factor. Nat Med 2013 ; 19: 1281-7.

49. Torisu K, Singh KK, Torisu T, et al. Intact endothelial autophagy is required to maintain vascular lipid homeostasis. Aging Cell 2016; 15 : 187-91.
50. Jeffery TK, Morrell NW. Molecular and cellular basis of pulmonary vascular remodeling in pulmonary hypertension. Prog Cardiovasc Dis $2002 ; 45: 173$ 202

51. Lee SJ, Smith A, Guo L, et al. Autophagic protein LCZB confers resistance against hypoxia-induced pulmonary hypertension. Am J Respir Crit Care Med $2011 ; 183: 649-58$.

52. Long L, Yang X, Southwood M, et al. Chloroquine prevents progression of experimental pulmonary hypertension via inhibition of autophagy and lysosomal bone morphogenetic protein type II receptor degradation. Circ Res 2013; 112 : 1159-70

53. Zheng $\mathrm{YH}$, Tian C, Meng $\mathrm{Y}$, et al. Osteopontin stimulates autophagy via integrin/CD44 and p38 MAPK signaling pathways in vascular smooth muscle cells. J Cell Physiol $2012 ; 227$ : 127-35.

54. Rouer M, Xu BH, Xuan HJ, et al. Rapamycin limits the growth of established experimental abdominal aortic aneurysms. Eur J Vasc Endovasc Surg 2014 ; $47: 493-500$

55. Ramadan A, Wheatcroft MD, Quan A, et al. Effects of long-term chloroquine administration on the natural history of aortic aneurysms in mice. (an J Physiol Pharmacol $2015 ; 93: 641-8$.

56. Morel $\varepsilon$. La formation de l'autophagosome : un nouveau défi pour le biologiste cellulaire. Med Sci (Paris) 2017 ; $33: 217-20$.

57. Vigié $P$, Camougrand N. Mitophagie et contrôle qualité des mitochondries. Med Sci (Paris) $2017 ; 33: 231-7$

\section{TIRÉS À PART}

C.M. Boulanger e u'est-ce que la guérison? Des réponses, il y en a. De toutes sortes et de tout temps. Chacun y va de son savoir religieux, scientifique, médical... Et de quoi est-on supposé guérir ? D’un symptôme, d'une douleur, d'une maladie, d'une répétition mortifère, d'un destin mélancolique ? Pour la psychanalyse, la guérison s'insère dans un système imaginaire et a, comme point de mire, un idéal. «La guérison, c'est une demande... » précise Lacan. Les auteurs nous invitent ici à découvrir, au-delà du semblant et à partir

Qu'est-ce que la guérison pour la psychanalyse?
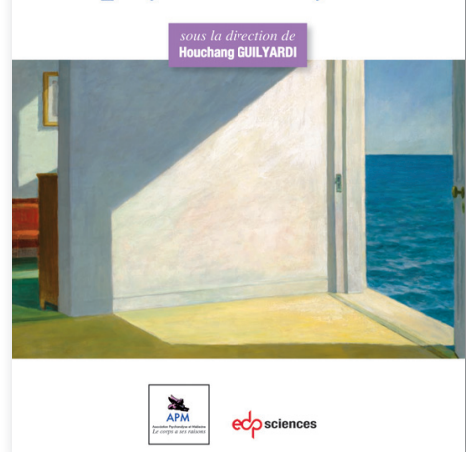

de la clinique, les liens entre guérison et vérité du sujet.

Comité éditorial de l'Association Psychanalyse et Médecine (APM) : Martine Dombrosky, Sophie Dunoyer de Segonzac, Houchang Guilyardi, Josette Olier, Betty Testud

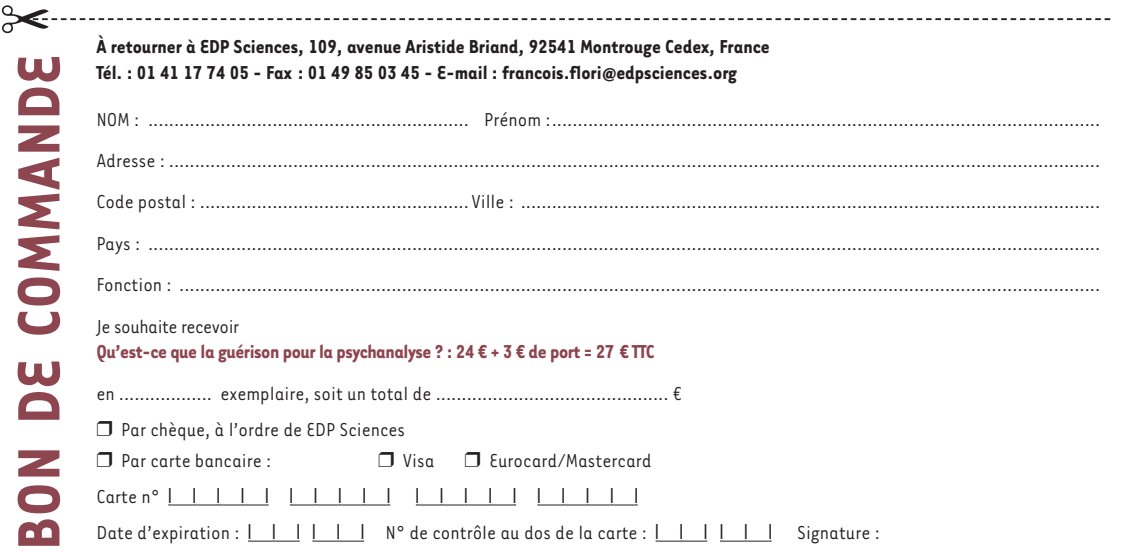

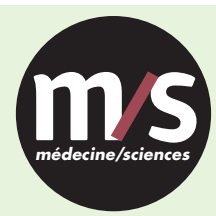

Tarifs d'abonnement $\mathrm{m} / \mathrm{s}-2017$

Abonnez-vous à médecine/sciences
$>$ Grâce à $m / s$, vivez en direct les progrès des sciences biologiques et médicales

Bulletin d'abonnement page 362 dans ce numéro de $\mathrm{m} / \mathrm{s}$

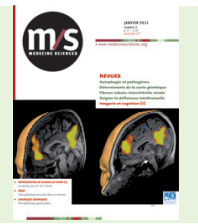

